\title{
Adenosine deaminases that act on RNA induce reproducible changes in abundance and sequence of embryonic miRNAs
}

\author{
Cornelia Vesely, ${ }^{1,4}$ Stefanie Tauber, ${ }^{2,3,4}$ Fritz J. Sedlazeck, ${ }^{2,3}$ Arndt von Haeseler, ${ }^{2,3}$ \\ and Michael F. Jantsch ${ }^{1,5}$ \\ ${ }^{1}$ Department of Chromosome Biology, Max F. Perutz Laboratories, University of Vienna, A-1030 Vienna, Austria; ${ }^{2}$ Center for Integrative \\ Bioinformatics Vienna, Max F. Perutz Laboratories, University of Vienna, Medical University of Vienna, A-1030 Vienna, Austria; \\ ${ }^{3}$ University of Veterinary Medicine, A-1030 Vienna, Austria
}

\begin{abstract}
Adenosine deaminases that act on RNA bind double-stranded and structured RNAs and convert adenosines to inosines by hydrolytic deamination. Inosines are recognized as guanosines, and, hence, RNA editing alters the sequence information but also structure of RNAs. Editing by ADARs is widespread and essential for normal life and development. Precursors of miRNAs are abundantly edited by ADARs, but neither the abundance nor the consequences of miRNA editing has been firmly established. Using transgenic mouse embryos that are deficient in the two enzymatically active editing enzymes ADAR and ADARBl, we compare relative frequencies but also sequence composition of miRNAs in these genetically modified backgrounds to wild-type mice by "next-generation sequencing." Deficiency of ADARBI leads to a reproducible change in abundance of specific miRNAs and their predicted targets. Changes in miRNA abundance seem unrelated to editing events. Additional deletion of ADAR has surprisingly little impact on the mature miRNA repertoire, indicating that miRNA expression is primarily dependent on ADARBI. A-to-G transitions reflecting A-to-I editing events can be detected at few sites and at low frequency during the early embryonic stage investigated. Again, most editing events are ADARBI-dependent with only few editing sites being specifically edited by ADAR. Besides known editing events in miRNAs, a few novel, previously unknown editing events were identified. Some editing events are located to the seed region of miRNAs, opening the possibility that editing leads to their retargeting.
\end{abstract}

[Supplemental material is available for this article.]

Adenosine deaminases that act on RNAs (ADARs) are doublestranded RNA binding proteins that hydrolytically deaminate adenosines to inosines in double-stranded RNA structures. This process is known as A-to-I editing. In mammals, three ADARs designated as ADAR (also known as ADAR1), ADARB1 (also known as ADAR2), and ADARB2 (also known as ADAR3) are found. Of these, the brain-specific ADARB2 is considered to be inactive because no enzymatic activity could be detected either in vitro, or in vivo (Nishikura 2010). Substrates for ADARs are double-stranded or structured RNAs that are bound by the double-stranded RNA binding domains (dsRBDs) found in ADARs. dsRBDs show little sequence specificity but can recognize specific structures such as terminal loops (Stefl et al. 2010; Barraud and Allain 2012). Consistently, ADARs can edit promiscuously but also very site-specifically, depending on the substrate. Because inosine (I) base-pairs with cytosine, it is interpreted as guanosine $(\mathrm{G})$ by the cellular machineries. Thus, editing leads to protein recoding in some genes (Gallo and Locatelli 2012) but can also alter the secondary structure and stability of an RNA.

Editing is an essential process, because Adarb1 $1^{-1-}$ mice die around day 20 after birth due to epileptic seizures. The phenotype is caused by the lack of editing of glutamate receptor B (GRIA2, also

\footnotetext{
${ }^{4}$ These authors contributed equally to this work.

${ }^{5}$ Corresponding author

E-mail Michael.Jantsch@univie.ac.at

Article published online before print. Article, supplemental material, and publication date are at http://www.genome.org/cgi/doi/10.1101/gr.133025.111. Freely available online through the Genome Research Open Access option.
}

known as GluR-B), which is a substrate for ADARB1 (Brusa et al. 1995). ADAR-deficient mice already die around embryonic day 12 showing rapid liver disintegration and hematopoietic defects (Hartner et al. 2004). It was shown that ADAR plays an essential role in hematopoietic stem cell maintenance and suppresses interferon signaling (Hartner et al. 2009). However, the exact molecular basis for the severe embryonic phenotype remains elusive.

Currently, a few dozens of protein-coding mRNA targets are known, although their list is ever increasing. Interestingly, the majority of protein-coding substrates for editing have been identified in nervous tissue (Sommer et al. 1991; Brusa et al. 1995; Burns et al. 1997; Hoopengardner et al. 2003; Ohlson et al. 2007). However, the majority of A-to-I editing events do not affect coding, but noncoding sequences. The most abundant editing events occur in SINEs. When multiple of these elements are found in inverted orientation within the same RNA, base-pairing between the elements produces double-stranded structures that serve as editing substrates (Levanon et al. 2004; Li et al. 2009). In humans, thousands of mRNAs showing edited SINEs have been identified. The consequences of these editing events are still under debate. Potentially, editing in 3' UTRs can affect transport, localization, stability, or translation of substrate RNAs.

Another class of noncoding RNAs targeted by ADARs are the stem-loops formed by repetitive regions and miRNA precursors (Wu et al. 2011). The primary miRNA transcript folds into an imperfect double-stranded structure and is subsequently cleaved by DROSHA-DGCR8 into a 60-70-nt-long premature miRNA (premiRNA). After export into the cytoplasm via EXPORTIN 5/RAN- 
GTP, the pre-miRNA is processed by DICER1, resulting in a 19-24nt-long mature miRNA. The mature miRNA finally is incorporated into RISC, which hybridizes to partially complementary sites in the 3' UTR of target mRNAs. This can either result in a translational block or in degradation of target mRNAs (Bartel 2004).

Editing of miRNA precursors can affect miRNA processing steps or lead to changes in the target specificity of the edited mature miRNA. Examples of miRNAs that are either not processed by DROSHA or DICER1 upon A-to-I editing have been demonstrated (Yang et al. 2006; Kawahara et al. 2007a). In rare cases, editing even increases the processing efficiency of the miRNA (Kawahara et al. 2008). Moreover, in those cases in which editing occurs in the recognition or 5 '-seed sequence (nucleotides 2-8), editing can lead to retargeting. One remarkable example in which editing in the 5 '-seed region changes target specificity is miR-376. The edited form of this miRNA was shown to specifically target a different set of genes (Kawahara et al. 2007b). Besides this striking effect of ADARs on miRNA targeting, it was also shown that ADARs can affect miRNAs even in the absence of editing, simply by binding the double-stranded structure (Heale et al. 2009). If this is a common phenomenon, one can expect many more miRNAs than the known edited ones to be affected by the lack of ADARs.

To precisely determine the impact of RNA editing on the miRNA repertoire of an organism, we have used next-generation sequencing to compare miRNA pools derived from embryonic mice lacking ADARB1, or ADAR and ADARB1, with those from wild-type embryos.

\section{Results}

\section{Abundance of mature miRNAs in embryos deficient in ADARs}

Editing of miRNAs by ADARs can interfere with their proper processing by either DROSHA or DICER1. Moreover, the presence of inosines in miRNAs and their precursors can even lead to their degradation by Tudor-SN (Yang et al. 2006; Kawahara et al. 2007a, 2008). Interestingly, ADARs can even interfere with miRNA maturation in the absence of editing, presumably by binding to miRNA precursors (Heale et al. 2009). We thus hypothesized that miRNAs that are normally impaired in processing by editing will get enriched in the pool of mature miRNAs in the absence of editing. Vice versa, miRNAs that get more efficiently processed upon editing should be reduced in the absence of ADAR and ADARB1 (Kawahara et al. 2008). To determine sequence variation but also changes in relative abundance induced by RNA editing, we isolated small RNAs (19-25 nt) from wild-type, Adarb $^{-1-}$, and Adar ${ }^{-1-}$, Adarb1 ${ }^{-1-}$ mouse embryos and subjected them to deep sequencing. (The Adarb1 $1^{-1-}$ and Adar $^{-1-}$, Adarb $1^{-l-}$ mouse strains were a kind gift of the laboratory of Peter Seeburg, MPI Heidelberg [Higuchi et al. 2000; Hartner et al. 2004].) Since Adar ${ }^{-1-}$ embryos die at embryonic day 12.5 (E12.5) and to avoid any sex-specific differences, E11.5 female embryos were chosen. Wherever possible, different genotypes were picked from siblings resulting from heterozygous crosses. For each genotype and biological replicate, one lane on an Illumina GAIIx was used. On average, 25 million total reads were obtained per lane (see Supplemental Table 1). Of these, $\sim 40 \%$ were mappable to annotated mature miRNA sequences (release 16 of miRBase) (Kozomara and Griffiths-Jones 2011; see Methods). We call a read mappable to a miRNA if it has at most two mismatches. Three independent biological replicates of each genotype were examined for all analyses. After mapping against mature mouse miRNAs using an inhouse next-generation sequencing mapper, miRNA reads were normalized to total mappable miRNA read counts per sequencing lane. This was done to correct for the fact that sequencing depth and percentage of miRNAs per total reads varied between biological replicates (see Supplemental Table 1).

Of the 1055 mature miRNAs annotated in miRBase, we detected on average 993 in the three biological replicates, allowing a maximum of two mismatches and considering a coverage of at least 10 reads per miRNA and run. Among these, only a small set of 14 miRNAs accounted for $47 \%$ of the mappable reads (Supplemental Fig. 1). This group of most abundant miRNAs was composed of the same members in all replicates and in all three genotypes, with mmu-miR-199a-3p being most abundant. Of the most abundant miRNAs, mmu-miR-124 consistently showed a strong reduction in coverage to $59 \%$ in both Adarb $^{-1-}$ and Adar $^{-1-}$, Adarb1 ${ }^{-1-}$ mice. Mmu-miR-124 is not reported to be edited, and our sequence data also fail to detect editing in the mature miRNA. However, it contains two adenosines (one in the $-5 p$ and one in the $-3 p$ sequence) that match the reported editing site preferences of ADAR and ADARB1 reasonably well (Eggington et al. 2011). Editing of the corresponding pri-miRNA or pre-miRNA can therefore not be excluded.

However, the known edited miRNAs in this set of most abundant miRNAs, namely, mmu-miR-130b, -17, and -143, showed similar abundance in wild-type and knockout individuals (Fig. 1).

When further investigating changes in miRNA abundance in different genotypes, we restricted our analysis to miRNAs with a coverage of at least 300 reads, to stay biologically relevant. Relative abundance to wild-type expression was calculated for all 418 miRNAs that were above this threshold. Of these, $4 \%$ of miRNAs were down-regulated by at least 1.3 -fold in both knockout genotypes, and $35 \%$ and $32 \%$ of miRNAs were at least 1.3 -fold upregulated in $A d a r b 1^{-1-}$ and $A d a r^{-1-}, A_{d a r b 1^{-1-}}$ mice, respectively. Thus, the effect of deficiency of ADARs led mostly to an upregulation of mature miRNA sequences. This also fits previous

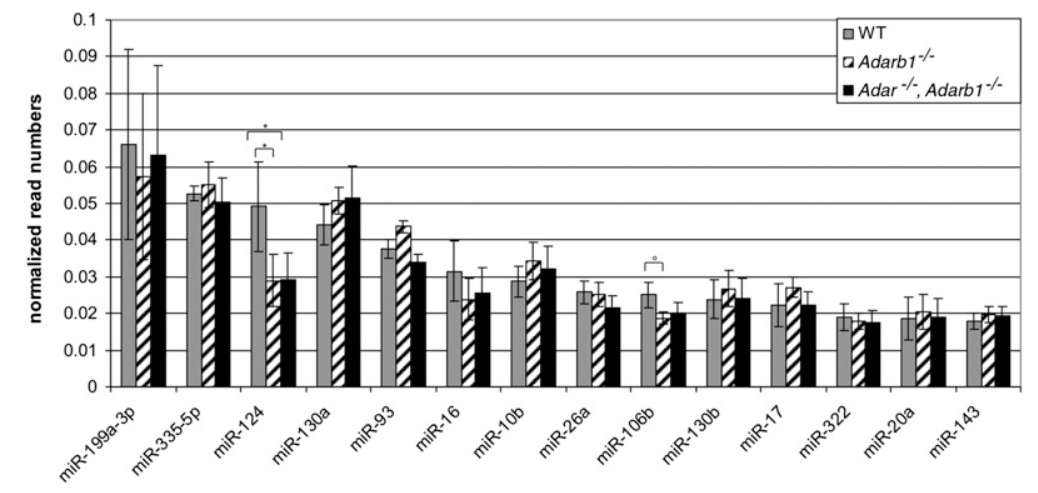

Figure 1. A small set of highly abundant miRNAs found in all biological replicates and all genotypes. Normalized read numbers of the 14 most highly abundant miRNAs in wild-type, Adarb1 $1^{-1-}$, and Adar ${ }^{-1-}$, Adarb $1^{-1-}$ mice are shown $(n=3)$. Of these miRNAs, only mmu-miRNA-130b, -17 , and -143 have previously been shown to be edited (Yang et al. 2006; Landgraf et al. 2007; Kawahara et al. 2008). (*) Student's $t$-test, $P<0.07 ;\left(^{\circ}\right)$ Student's $t$-test, $P<0.1$. 
findings in which more examples where editing leads to a block in miRNA processing were found than cases where editing enhanced processing. A complete list of consistently up-regulated and down-regulated miRNAs is shown in Supplemental Table 2.

\section{Changes in edited miRNAs in mice deficient in ADARs}

According to previous findings, quantitative changes in miRNA abundances dependent on ADARs were primarily expected in edited miRNAs. Moreover, since both ADARs can potentially act on pri-miRNAs, quantitative changes were expected to be more dramatic in $\mathrm{Adar}^{-1-}$, Adarb1 ${ }^{-1-}$ double-knockout embryos than in Adarb1 $1^{-1-}$ single-knockout samples. However, individual miRNA abundances were similar for both single- and double-knockout embryos when compared with wild-type embryos, suggesting that a large fraction of observed changes results from ADARB1 deficiency (Fig. $2 \mathrm{~A}, \mathrm{~B})$. More precisely, when miRNAs with a significant, at least 1.3-fold, up-regulation or down-regulation in $\mathrm{Adar}^{-/}$, Adarb $1^{-1-}$ double-knockout embryos were considered, at least $52 \%$ showed the same significant change in Adarb $1^{-/-}$ mutant embryos. Of the remaining $48 \%$, only a single miR-30b showed an opposing trend (up-regulation in $\mathrm{Adar}^{-1-}$, Adarb $^{-1-}$, down-regulation in Adarb $1^{-1-}$ embryos), while the rest showed minor alterations that were nonsignificant in embryos lacking ADARB1 only (Supplemental Table 2).

The same was true when only miRNAs that were proven to be edited were considered (Fig. 2C). In addition, among edited miRNAs, a larger proportion of miRNAs was up-regulated in the absence of either one or two ADARs, while a smaller fraction of miRNAs was downregulated. More precisely, only $2 \%$ of the known edited miRNAs showed an at least 1.3-fold down-regulation in both knockout genotypes, whereas $36 \%$ and $33 \%$ were up-regulated by at least 1.3 -fold in the Adarb1 $1^{-1-}$ and Adar $^{-1-}$, Adarb1 $1^{-1-}$ mice, respectively. However, the majority of edited miRNAs did not show any significant difference in abundance compared with wild-type levels, indicating that editing or the presence of ADARs does not affect their processing. Moreover, in some cases, changes in abundance were similar or even higher in ADARB1 single-mutant mice than in the double-mutant embryos (Fig. 2B). Table 1 lists edited miRNAs with a consistent up-regulation or down-regulation in at least one of

B
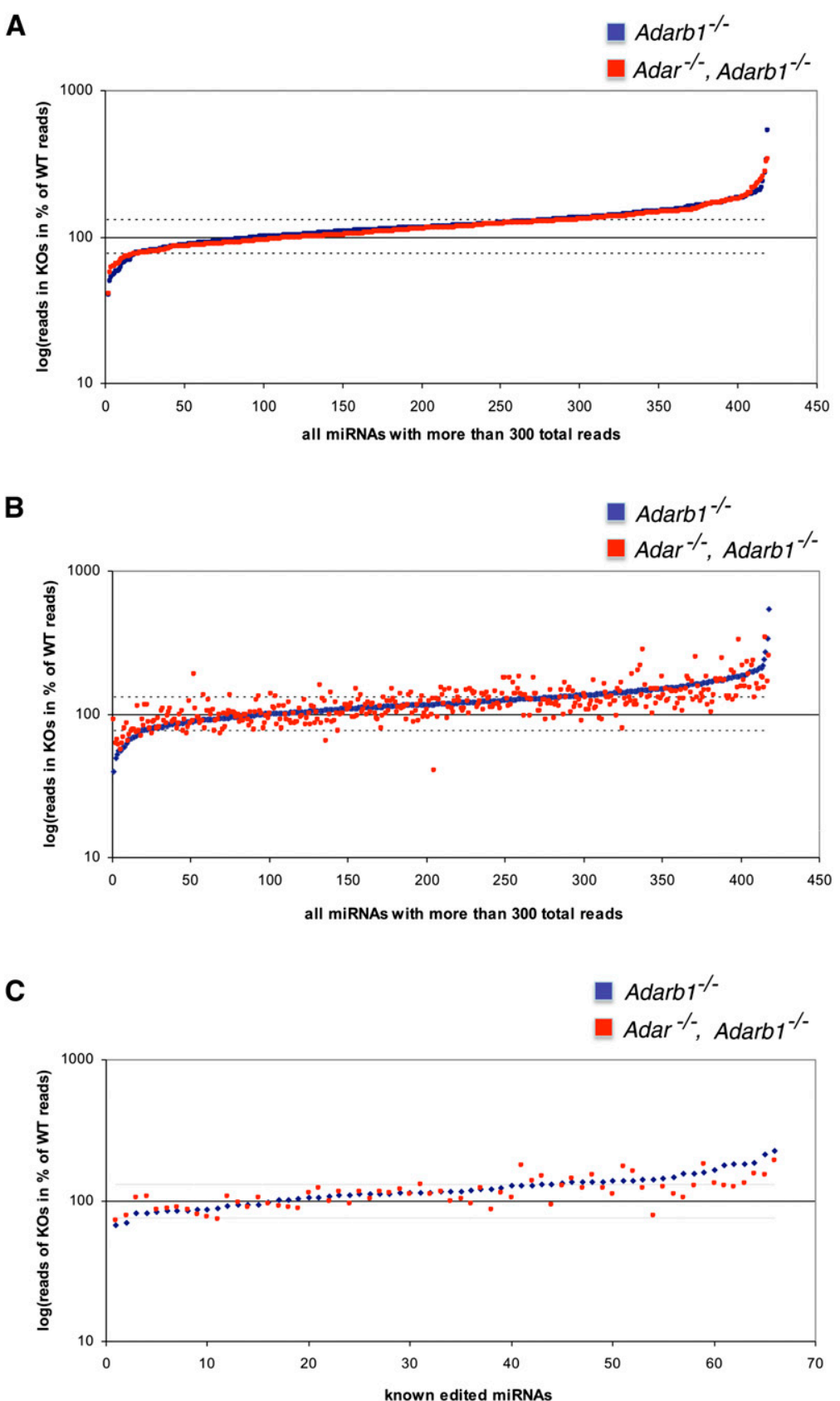

Figure 2. Edited and unedited miRNAs show a similar distribution of relative abundance in Adarb $1^{-1-}$ and Adar $^{-1-}$, Adarb $1^{-1-}$ embryos. (A) miRNAs with a read depth $>300$ were sorted according to their relative abundance to wild type (displayed as \% of wild-type abundance) in both KO genotypes and plotted on a half-logarithmic scale. miRNAs in Adarb $^{-1-}$ (blue) and Adar ${ }^{-1-}$, Adarb $1^{-1-}$ (red) mice. Wild-type expression level is represented by 100 , and a threshold of 1.3 -fold deregulation is marked by dashed lines. (B) miRNAs shown in $A$ are sorted by their abundance in the Adarb $1^{-1-}$ embryos. (C) miRNAs known from literature to be A-to-l edited were sorted according to their relative abundance in the Adarb1 $^{-1-}$ embryos (displayed as \% of wild-type abundance). miRNAs in Adarb1 $1^{-1}$ (blue) and $\mathrm{Adar}^{-1-}$, Adarb $^{-1-}$ (red) mice. Wild-type expression level is represented by 100 , and a threshold of 1.3 -fold deregulation is marked by dashed lines.

the mutant genotypes. For example, mmu-miR-532-5p, -142-5p, $-450 \mathrm{~b}-3 \mathrm{p}$, and $376 \mathrm{~b}$ are consistently up-regulated in the ADARB1deficient mice but unaffected or much less affected in the doubleknockout mice (Table 1). Together, this demonstrates that miRNA abundance and therefore miRNA processing are mainly influenced

\section{Genome Research www.genome.org}


ADAR- and ADARB1-induced changes in miRNAs

Table 1. Edited miRNAs that are consistently up-regulated or down-regulated

\begin{tabular}{|c|c|c|c|c|c|}
\hline & $\begin{array}{l}\text { Fold change } \\
\text { in Adarb1 } 1^{-/-}\end{array}$ & $P$-value & $\begin{array}{l}\text { Fold change in } \\
\text { Adar }^{-/-}, \text {Adarb } 1^{-/-}\end{array}$ & $P$-value & Reference for editing \\
\hline \multicolumn{6}{|c|}{ Down-regulated edited miRNAs } \\
\hline let-7g & -1.5 & 0.01 & -1.39 & 0.105 & Kawahara et al. 2008; Chiang et al. 2010 \\
\hline miR-3099 & -1.15 & 0.19 & -1.31 & 0.03 & Chiang et al. 2010 \\
\hline $\operatorname{miR}-376 \mathrm{c}$ & -1.14 & 0.362 & -1.35 & 0.07 & Kawahara et al. 2008; Chiang et al. 2010 \\
\hline \multicolumn{6}{|c|}{ Up-regulated edited miRNAs } \\
\hline miR-219-3p* & 2.41 & 0.099 & 1.53 & 0.067 & Chiang et al. 2010 \\
\hline miR-450b-5p & 2.08 & 0.025 & 1.51 & 0 & Landgraf et al. 2007 \\
\hline miR-106a & 1.86 & 0.062 & 1.55 & 0.223 & Kawahara et al. 2008 \\
\hline miR-450b-3p & 1.81 & 0.031 & 1.25 & 0.111 & Landgraf et al. 2007 \\
\hline miR-376b & 1.79 & 0.01 & 1.29 & 0.198 & Kawahara et al. 2008 \\
\hline miR-19b & 1.58 & 0.167 & 1.81 & 0 & Landgraf et al. 2007 \\
\hline miR-379* & 1.55 & 0.052 & 1.27 & 0.186 & Chiang et al. 2010 \\
\hline miR-532-5p & 1.55 & 0.052 & 1.06 & 0.378 & Kawahara et al. 2008 \\
\hline $\operatorname{miR}-144^{*}$ & 1.55 & 0.001 & 1.47 & 0.076 & Blow et al. 2006 \\
\hline miR-142-5p & 1.48 & 0.044 & 1.11 & 0.29 & Yang et al. 2006 \\
\hline miR-381 & 1.44 & 0.073 & 1.24 & 0.052 & Chiang et al. 2010 \\
\hline miR-133a* & 1.34 & 0.087 & 1.52 & 0.062 & Blow et al. 2006; Kawahara et al. 2008 \\
\hline miR-17* & 1.32 & 0.064 & 1.29 & 0.193 & Landgraf et al. 2007 \\
\hline miR-503* & 1.13 & 0.309 & 1.3 & 0.028 & Kawahara et al. 2008 \\
\hline
\end{tabular}

The fold changes marked in bold are consistent over all three replicates (Student's $t$-test, $P<0.1$ ). Only miRNAs with a minimum of 1.3 -fold up-regulation or down-regulation were considered. $\left({ }^{*}\right)$ A miRNA expressed at low levels relative to the miRNA on the opposite arm in miRBase release 18.

by ADARB1 and that an additional loss of ADAR has only a minor effect on expression of mature miRNAs.

\section{Editing-independent changes in miRNA abundance}

Many miRNAs that are apparently not edited are strongly upregulated or down-regulated in an ADARB1 or ADAR and ADARB1-deficient background (Fig. 2; Supplemental Table 2). We therefore wondered whether the most deregulated miRNAs would harbor possible editing sites close to their DROSHA or DICER1 cleavage site but outside the mature miRNA so that editing would remain undetected in deep-sequencing approaches that focus on the mature product. Such editing sites adjacent to processing sites could lead to binding of ADARs but also induce structural changes due to editing that could both interfere with miRNA processing.

Within a range of $4 \mathrm{nt}$ upstream of and downstream from the respective processing sites, we screened for putative editing sites harboring adenosines with a flanking 5' $\mathrm{U}$ and a G, C, or A as the 3' nucleotide, which reflects good target sites for both ADARs (Supplemental Table 2; Eggington et al. 2011). Twenty-five miRNAs that are consistently highly up-regulated in one or both knockout genotypes (five miRNAs overlap in Adarb1 ${ }^{-/-}$and Adar $^{-1-}$, Adarb $1^{-l-}$ mice) were analyzed. Of these, nine and eight miRNAs have a preferential editing site near the DROSHA or the DICER1 cleavage site, respectively. Similarly, of 15 down-regulated miRNAs (five miRNAs overlap in Adarb1 $1^{-1-}$ and $\mathrm{Adar}^{-1-}$, Adarb $^{-1-}$ mice), three and eight possible editing sites were detected near the DROSHA or DICER1 cleavage sites, respectively (Supplemental Table 2).

However, when we screened for the presence of candidate editing sites in pri-miRNAs that were unaffected by ADARs, we could detect such sites at similar frequencies as in the deregulated sample. Moreover, a large group of highly up-regulated or downregulated miRNAs still lacks putative editing sites. Thus, it seems unlikely that editing is the major cause for the observed changes in miRNA abundances.
Changes in miRNA abundance affect potential target mRNAs

To test whether changes in miRNA levels dependent on ADARs have an impact on predicted target mRNAs, expression profiles of wild-type, Adarb1 ${ }^{-1-}$, and Adar $^{-1-}$, Adarb1 $^{-1-}$ female E11.5 embryos were analyzed on whole mouse genome microarrays. Putative target mRNAs were either picked from published data or, alternatively, selected by target predictions using TargetScan 5.2 (Friedman et al. 2009). Expression of these putative targets was compared in the two knockout genotypes relative to wild type. Indeed, a few known and experimentally validated targets of deregulated miRNAs showed altered expression in our microarray data. Similarly, when the first 20 hits of a TargetScan search were included in our analysis, a few more putative targets were found deregulated in the microarray analysis. A summary of miRNAs and their regulated targets is shown in Table 2. Interestingly, many of the miRNAs affected by loss of ADARs target mRNAs of membrane proteins (CNTN2:: mmu-miR-34b-5p and -34c; CELSR3:: mmumiR-30a-c/e; and EFNA3:: mmu-miR-210, -137, and -673-5p) (Hu et al. 2010). In the group of inversely regulated miRNA-mRNA pairs, also three previously reported, edited miRNAs and their targets were found. NEROD1 (neurogenic differentiation 1) is known to be targeted by mmu-miR-19b (Zhang et al. 2011), whereas the targets of the reported edited mmu-let-7g and $\mathrm{mmu}-$ miR-153, HOXA9 (homeobox A9), and SYN2 (synapsin II) are predictions of TargetScan. The predicted target for mmu-miR-153, SYN2, is also predicted to be targeted by mmu-miR-30a-c\&e as well as mmu-miR-32, all consistently up-regulated in the double knockout (Table 2). The fact that we were able to correlate the expression changes of some miRNAs in the knockout mice with the expression of potential target mRNAs underscores the importance of ADARs for mature miRNA homeostasis.

\section{Transitions dependent on ADARs in mature miRNAs}

A-to-I editing levels increase during development as shown for some specific substrates of ADARB1 (Wahlstedt et al. 2009). Thus, 
Table 2. miRNAs deregulated in mice deficient in ADARs and their corresponding targets

\begin{tabular}{|c|c|c|c|c|c|c|c|c|c|c|}
\hline miRNA & $\begin{array}{l}\text { miRNA fold } \\
\text { change in } \\
\text { Adarb1 } 1^{-/-}\end{array}$ & $P$-value & $\begin{array}{l}\text { mRNA fold } \\
\text { change in } \\
\text { Adarb1 } 1^{---}\end{array}$ & $P$-value & $\begin{array}{c}\text { miRNA } \\
\text { fold change } \\
\text { in } \text { Adar }^{-1-} \\
{\text { Adarb } 1^{-/-}}^{\prime}\end{array}$ & $P$-value & $\begin{array}{c}\text { mRNA } \\
\text { fold change } \\
\text { Adar }^{-/-} \\
{\text {Adarb } 1^{-/-}}^{-}\end{array}$ & $P$-value & $\begin{array}{c}\text { TargetScan } \\
\text { total } \\
\text { context } \\
\text { score }\end{array}$ & Target \\
\hline $\begin{array}{l}\text { let-7a } \\
\text { let-7c } \\
\text { let-7d } \\
\text { let-7g }\end{array}$ & $\begin{array}{l}-1.4 \\
-2.2 \\
-1.8 \\
-1.5\end{array}$ & $\begin{array}{c}0.011 \\
2.1 \times 10^{-4} \\
0.003 \\
0.010\end{array}$ & 2.3 & $2 \times 10^{-6}$ & $\begin{array}{l}-1.3 \\
-1.6 \\
-1.6 \\
-1.4\end{array}$ & $\begin{array}{l}0.089 \\
0.106 \\
0.056 \\
0.105\end{array}$ & 1.8 & $2 \times 10^{-4}$ & $\begin{array}{l}-0.18 \\
-0.18 \\
-0.18 \\
-0.18\end{array}$ & HOXA9 ${ }^{a}$ \\
\hline miR-124 & -1.7 & $3.7 \times 10^{-7}$ & 1.5 & $7 \times 10^{-3}$ & -1.6 & 0.007 & 2.5 & $6 \times 10^{-15}$ & -0.74 & TRIB3 \\
\hline miR-190 & 1.9 & 0.156 & 1.0 & $7 \times 10^{-1}$ & 2.2 & 0.041 & -5.8 & $2 \times 10^{-6}$ & -0.51 & ZC3H6 \\
\hline miR-19b & 1.6 & 0.167 & -2.4 & $2 \times 10^{-5}$ & 1.8 & $4.1 \times 10^{-4}$ & -1.8 & $3 \times 10^{-4}$ & -0.65 & NEUROD 1 \\
\hline miR-673-5p & 1.2 & 0.442 & 1.2 & $5 \times 10^{-2}$ & -2.4 & 0.093 & 2.3 & $5 \times 10^{-7}$ & -0.27 & \\
\hline miR-137 & 1.1 & 0.393 & 1.2 & $5 \times 10^{-2}$ & -1.5 & 0.011 & 2.3 & $5 \times 10^{-7}$ & -0.22 & EFNA3 $^{\mathrm{C}}$ \\
\hline miR-210 & 1.4 & 0.199 & 1.2 & $5 \times 10^{-2}$ & -1.3 & 0.005 & 2.3 & $5 \times 10^{-7}$ & -0.18 & \\
\hline miR-30a & 1.3 & 0.179 & -1.3 & $6 \times 10^{-2}$ & 1.4 & 0.021 & -2.5 & $4 \times 10^{-8}$ & -1.57 & CELSR3 \\
\hline miR-30b & -1.1 & 0.042 & & & 1.9 & 0.054 & & & & $\&$ \\
\hline miR-30c & 1.3 & 0.229 & -1.3 & $9 \times 10^{-4}$ & 1.8 & 0.047 & -4.7 & $5 \times 10^{-11}$ & -0.18 & SYN2 \\
\hline miR-30e & 1.2 & 0.269 & & & 1.4 & 0.044 & & & & \\
\hline miR-32 & 1.4 & 0.265 & -1.3 & $9 \times 10^{-4}$ & 1.7 & 0.054 & -4.7 & $5 \times 10^{-11}$ & -0.75 & SYN2 \\
\hline miR-153 & 1.4 & 0.179 & -1.3 & $9 \times 10^{-4}$ & 1.3 & 0.020 & -4.7 & $5 \times 10^{-11}$ & -0.15 & SYN2 \\
\hline miR-335-3p & 1.9 & 0.081 & -4.6 & $8 \times 10^{-3}$ & 1.9 & 0.095 & -5.2 & $4 \times 10^{-3}$ & -0.89 & STXBP5L \\
\hline miR-34b-5p & 1.7 & 0.059 & -2.4 & $3 \times 10^{-8}$ & 1.7 & 0.020 & -2.3 & $1 \times 10^{-5}$ & -0.66 & CNTN2 \\
\hline $\mathrm{miR}-34 \mathrm{c}$ & 1.3 & 0.031 & -2.4 & 3. $\times 10^{-8}$ & 1.4 & 0.021 & -2.3 & $1 \times 10^{-5}$ & -0.66 & CNTN2 \\
\hline
\end{tabular}

Either up-regulated or down-regulated miRNAs, for which inversely regulated targets were found in the microarray analysis, are listed. (Underlined) Known edited miRNAs. Significant down- or up-regulation is marked in bold.

ashen et al. 2008.

${ }^{\mathrm{b}}$ Zhang et al. 2011.

'Hu et al. 2010.

we expected only a low editing frequency of mature miRNAs in embryos at E11.5. Still, we analyzed the amount of A-to-G transitions compared with all 11 other possible transition types in mature miRNAs in wild type and the two knockout genotypes. Lack of ADARs should lead to a decrease of A-to-G changes in mature miRNAs. This change should be more pronounced in the $\mathrm{Adar}^{-1-}$, Adarb $1^{-1-}$ double-mutant embryos than in single Adarb $^{-1-}$ embryos.

To allow detection of up to two editing events, the mapping algorithm allowed for a maximum of two mismatches to known miRNAs. Furthermore, we just took reads into account that mapped $3^{\prime}$ to $5^{\prime}$ on the annotated miRNA list. The cloning protocol for miRNAs is strand-specific. Reads that mapped to the reverse complement strand were ignored because they either result from nonannotated novel miRNAs originating from the $5 \mathrm{p}$ - or $3 \mathrm{p}$ - sequence, or might be the result of erroneous mapping.

Reads were aligned to the best matching miRNAs. The number of mappable reads per miRNA was counted, whereas weighting was applied when there were several miRNAs with equal best alignment scores. Additionally, the number $(0,1$, or 2$)$ of mismatches per read and type of nucleotide transition was recorded for each position in the aligned reads. This allowed the generation of a tabular listing of all positions of transitions for all three genotypes and all replicates. Frequently, the last 2 or 3 bases are trimmed before mapping to avoid the lower quality of base calls toward the end of a read. We considered this step unnecessary because our mapping algorithm computes a local optimal pairwise se- quence alignment (Smith and Waterman 1981). Therefore, the algorithm automatically ignores bases that differ between a read and the reference toward the end of the read.

The frequency of each of the 12 possible nucleotide transitions was normalized by the sum of all transitions in all alignable reads in each replicate. By doing so, we were able to compare the overall nucleotide transition profile between the three genotypes. We observed all 12 possible nucleotide transitions in the sequenced mature miRNAs to different extents (Fig. 3). The number of A-to-G transitions, which likely result from A-to-I editing, was reduced to the same extent in both ddarb1 $^{-1-}$ and Adar $^{-1-}$, Adarb $1^{-1-}$ embryos. In addition, the reduction of A-to-G transitions was statistically significant in all reads. Thus, as already observed for the effect on miRNA abundance, ADARB1 seems to have the major impact on the amount of A-to-I editing within those regions of the miRNA precursors that eventually give rise to mature

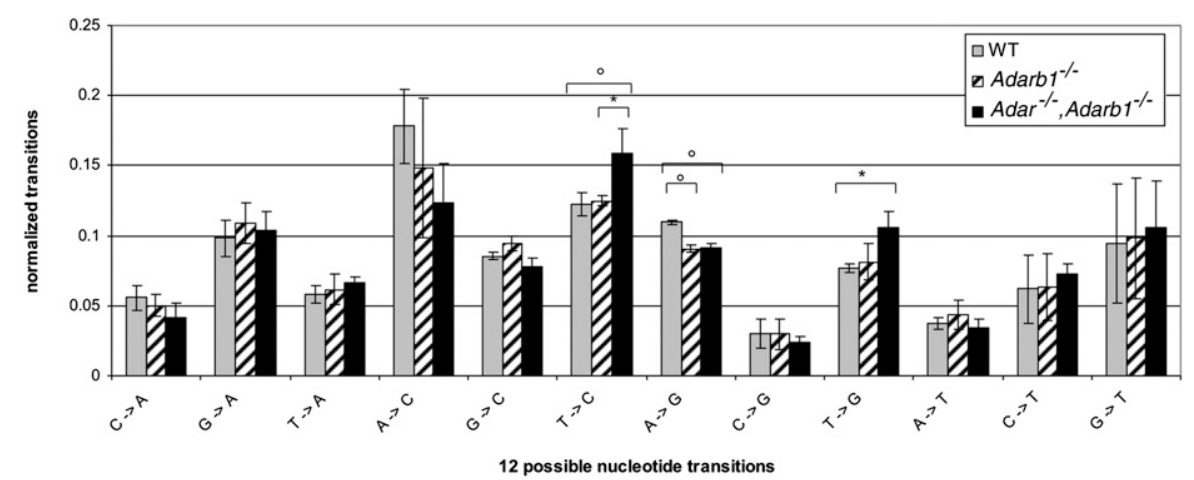

Figure 3. A-to-G transitions drop significantly in mice deficient in ADARs. All 12 possible nucleotide transitions in the miRNA reads were summed up and normalized to total transition numbers in all three replicates. $\left(^{*}\right)$ Student's $t$-test, $P<0.04 ;\left(^{\circ}\right)$ Student's $t$-test, $P<0.1$. 
miRNAs. Some transitions occurred to an even higher extent than the A-to-G transitions, but most of those showed no significant genotype-specific variation. However, T-to-C and T-to-G transitions, possibly resulting from U-to-C and U-to-G transitions at the RNA level, were significantly increased in the backgrounds deficient in ADARs. This observation remains to be analyzed further, because no biological mechanism is known for these kinds of substitutions. However, a recent study has also described abundant occurrence of non-canonical base transitions, raising the possibility of novel, unknown editing mechanisms (Li et al. 2011).

\section{Novel editing events in mature mouse miRNAs}

Despite the low editing levels at E11.5, we were still able to detect novel A-to-I editing events in our data set. The identification of editing events was done as follows. In each biological replicate, A-to-G transitions were recorded and counted for individual positions in all miRNAs for each genotype. Given an A in the mature miRNA sequence from miRBase, an editing event is called significant when more G's are observed than expected just due to sequencing error and experimental noise. These events were identified by means of a $\chi^{2}$ test (also see Methods). Significant events were computed separately for each biological replicate and genotype. Only those events for which a significant reduction in A-to-G transitions was consistently observed in at least two out of three Adar $^{-1-}$, Adarb1 $1^{-/-}$double-knockout replicates but not in the wildtype samples were considered further.

This method yields a list of "significant editing events" per miRNA and position (e.g., see Supplemental Table 3). Thirty miRNAs showed a significant A-to-G change at a certain position in at least two out of three replicates (Supplemental Table 4). Some of the miRNAs detected by us showed a low read number and thus low coverage. We therefore restricted the number of significantly edited miRNAs to those with more than 100 mapped reads. This resulted in a list of 10 miRNAs (Table 3), of which four were already known to be edited (mmu-miR-378, -376b, -381, -3099). Within these known edited miRNAs, only miR-378 is edited outside of the seed region (nucleotides $2-8$ from the $5^{\prime}$ end of the mature sequence). In general, four out of the 10 edited miRNAs are edited within the seed sequence. Also, $47 \%$ of the 30 miRNAs found edited in at least two out of three replicates show editing within the seed region (Supplemental Table 3). This enrichment for editing in the seed region goes along with the second function of ADARs in the interaction with the miRNA pathway, namely, retargeting of mature miRNAs as already reported for the mmu-miR-376 cluster (Kawahara et al. 2007b). However, at the early developmental stage of our mice, the editing efficiency is, as expected, low. Editing efficiencies for the detected significant editing events were calculated as reads with A-to-G transitions in percent of total reads of the miRNA. By doing so, editing efficiency ranged from very low $(0.2 \%)$ to low editing $(6.3 \%)$ in the 10 edited miRNAs (Table 3 ). Additionally, we determined the editing efficiency in the Adarb $1^{-1-}$ and $\mathrm{Adar}^{-1-}, \mathrm{Adarb1}^{-1-}$ mutant genotypes. In six out of the 10 edited miRNAs, editing levels already dropped dramatically in the Adarb $1^{-1-}$ mice, hinting at preferential editing by ADARB1. Only four of the detected edited miRNAs showed a drop in editing only in the double knockout, suggesting that those miRNAs are ADAR substrates or targeted by both editing enzymes (Table 3 ). In fact, position 6 of mature hsa-miR-376b was already shown to be targeted by both ADAR and ADARB1 (Heale et al. 2009). The editing efficiency of two miRNAs (mmu-miR-1957 and -706) even increased in Adarb1 $1^{-1-}$ mice, suggesting that these miRNAs are potential substrates of ADAR, that are normally protected from editing by binding to ADARB1 (Table 3 ).

Although no strict target sequence motif has been found for ADARs, there are certain preferences for the bases upstream, downstream, and opposite the edited adenosines (Wong et al. 2001; Kawahara et al. 2008; Kleinberger and Eisenberg 2010; Eggington et al. 2011). Recently, the editing site preferences for human ADAR and ADARB1 were redefined. Upstream from the editing site $U>A>C>G$ is favored by both ADARs. Whereas ADAR prefers an $G>C \approx A>U$ as the base downstream from the edited $A$, and ADARB1 preferentially edits before a $\mathrm{G}>\mathrm{C}>\mathrm{U} \approx \mathrm{A}$ (Eggington et al. 2011). The nucleotide opposing the editing site is usually either a $\mathrm{C}$ or $\mathrm{U}$ and rarely an A or $\mathrm{G}$ (Wong et al. 2001). In eight of the 10 identified edited miRNAs, the editing site fulfills the "next neighborhood" criteria for preferential editing. Of these 10 edited miRNAs, six are newly identified in this study, while four miRNAs

Table 3. Significant editing events detected in at least two out of three replicates of miRNAs with more than 100 total reads

\begin{tabular}{|c|c|c|c|c|c|c|c|c|c|c|c|c|}
\hline miRNA & $\begin{array}{c}\text { Average } \\
\text { read } \\
\text { number }\end{array}$ & Position & Context & $\begin{array}{c}\% \text { editing } \\
\text { wt }\end{array}$ & $\pm S D$ & $\begin{array}{l}\text { \% editing } \\
{\text { Adarb } 1^{-I}}^{-}\end{array}$ & $\pm S D$ & $\begin{array}{c}\text { \% editing } \\
\text { Adar }^{-/-} \\
\text {Adarb1 }^{-\prime-}\end{array}$ & $\pm S D$ & $\begin{array}{l}\text { Editing in } \\
\text { number of } \\
\text { replicates }\end{array}$ & $\begin{array}{l}\text { Most likely } \\
\text { edited by } \\
\text { ADARB1 }\end{array}$ & $\begin{array}{l}\text { Edited by } \\
\text { ADAR or } \\
\text { both } \\
\text { enzymes }\end{array}$ \\
\hline $\operatorname{miR}-378^{a}$ & 58589 & 16 & CAG / U & 0.22 & 0.00 & 0.13 & 0.04 & 0.04 & 0.01 & 2 & $x$ & \\
\hline miR-376b $b^{b}$ & 13612 & 6 & UAG / U & 0.68 & 0.33 & 0.39 & 0.14 & 0.02 & 0.03 & 2 & & $x$ \\
\hline miR-1957 & 2704 & 15 & $\mathbf{U A U} / \mathbf{U}$ & 0.18 & 0.02 & 0.35 & 0.01 & 0.10 & 0.08 & 2 & & $x$ \\
\hline $\mathbf{m i R} \mathbf{3 8 1 ^ { a }}$ & 2206 & 4 & $\mathbf{U A C} / \mathbf{U}$ & 1.02 & 0.21 & 1.09 & 0.02 & 0.07 & 0.09 & 3 & & $x$ \\
\hline $\operatorname{miR}-467 d^{*}$ & 1145 & 9 & UĀC / C & 0.57 & 0.23 & 0.02 & 0.02 & 0.04 & 0.01 & 2 & $x$ & \\
\hline miR-3099 & 684 & 7 & UAG / C & 3.52 & 0.28 & 1.68 & 0.81 & 0.07 & 0.09 & 2 & $x$ & \\
\hline miR-706 & 228 & 20 & $\mathbf{A A} \mathbf{A} / \mathbf{U}$ & 6.25 & 3.75 & 9.68 & 6.98 & 0.00 & 0.00 & 2 & & $x$ \\
\hline miR-1186 & 199 & 16 & $\mathbf{A A C} / \mathbf{C}$ & 1.92 & 0.12 & 0.65 & 0.61 & 0.00 & 0.00 & 2 & $x$ & \\
\hline miR-3102-5p.2 & 196 & 18 & $\mathrm{GAG} / \mathrm{U}$ & 1.30 & 0.10 & 0.12 & 0.12 & 0.10 & 0.14 & 2 & $x$ & \\
\hline miR-703 & 156 & 4 & $\mathbf{A} \mathbf{A} \mathbf{A} / \mathbf{U}$ & 2.04 & 1.19 & 0.28 & 0.28 & 0.00 & 0.00 & 2 & $x$ & \\
\hline
\end{tabular}

The five miRNAs in bold have already been identified as edited mature miRNAs. The underlined A in the "Context" column marks the edited adenosine, the nucleotide after the slash is the editing site opposing base. Sequence contexts in bold are preferred sites for editing. The "Position" column lists the nucleotide position of the edited adenosine in the mature miRNA sequence. Positions in bold are located within the seed sequence of the miRNA (nucleotides 2-8). (*) A miRNA expressed at low levels relative to the miRNA on the opposite arm in miRBase release 18.

${ }^{\mathrm{a}}$ Chiang et al. 2010

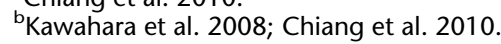


(mmu-miR-378, -376b, -381, -3099) had already been reported to be edited.

Of the four previously reported, edited miRNAs, three harbored a preferential editing site (mmu-miR-376b, -381, -3099), while a preferential editing site could be found in five out of six newly identified edited miRNAs (mmu-miR-1957, -467d*, 706, -1186, -703). Of these, mmu-miR-703 harbors the editing site within the seed sequence (Table 3).

\section{Discussion}

In this study, we have analyzed changes in abundance and sequence of miRNAs that occur in the absence of ADARs. We can show that lack of ADARs leads to a clear and reproducible change in abundance of certain miRNAs. Interestingly, changes in abundance can be found in both Adarb $1^{-1-}$ and Adar ${ }^{-1-}$, Adarb $1^{-1-}$ embryos. Changes in abundance vary from a 2.5 -fold down-regulation to a more than fourfold up-regulation in the absence of ADARs. Not only is there a stronger up-regulation than down-regulation in the absence of ADARs, but also the number of miRNAs that are up-regulated is significantly larger than those that are downregulated.

The general trend that lack of ADARs leads to an up-regulation of miRNAs is consistent with the previous observation that DROSHA or DICER1 cleavage can be antagonized by editing (Yang et al. 2006; Kawahara et al. 2007a, 2008). However, changes in miRNA abundance were also found in cases in which no editing had been detected or would even be expected based on the absence of candidate editing sites close to the processing sites. However, the recent finding that ADARs can interfere with miRNA processing even in the absence of editing is in good agreement with our finding (Heale et al. 2009). It might be that mere binding of ADARs can prevent efficient processing of miRNA precursors. In this context, it is interesting to note that the Adarb1 deletion allele used in this study truncates the deaminase domain but leaves the two dsRBDs untouched (Higuchi et al. 2000). Thus RNA binding of the remaining protein might be possible. However, it is not clear whether the remaining protein would stay stable or not.

Similarly, the Adar allele used here deletes exons 7-9 and thereby also deletes part of the deaminase domain and the last dsRBD. Again, it is not clear whether the remaining construct will form a stable protein that is able to bind RNA.

While both Adarb1 $^{-1-}$ and Adar ${ }^{-1-}$, Adarb $1^{-1-}$ mice show a large overlapping group of miRNAs that are affected in their abundance, either protein alone can also affect an individual subset of miRNAs in their abundance. This would suggest that some miRNA precursors are bound by ADARB1 while others are preferentially bound by ADAR, while a larger group of miRNA precursors is bound by either of the two enzymes. This is also consistent with the previous finding that specific adenosines in certain miRNAs can be edited by either enzyme as found for human miR-376a1 and miR-376a2 (Heale et al. 2009). However, in general, the impact of ADARB1 on miRNA processing seems to be more dramatic than that of ADAR. This, in turn, would suggest that the embryonic lethality observed in $\mathrm{Adar}^{-1-}$ mice is most likely not the effect of missing miRNAs.

The observed changes in miRNA abundance correlate nicely with a change in abundance of putative or proven target mRNAs. Microarray analysis allowed us to detect solid changes of a few mRNAs that are consistently up-regulated or down-regulated in mice deficient in ADARs. The fact that already proven targets such as HOXA9, NEUROD1, or EFNA3 were found inversely regulated to their regulatory miRNAs proves that the miRNA changes detected in the knockout mice are significant enough to exert consequences on target mRNAs. Moreover, the fact that some miRNAs and their targets can be found misregulated in the Adarb $1^{-1-}$ single knockout suggests that the observed effect is in direct correlation to the presence of ADARB1. Since the Adarb1 ${ }^{-1-}$ embryos are viable until $3 \mathrm{wk}$ after birth, no indirect, cell-death-related effect would be expected.

Some miRNAs and their putative target RNAs show a stronger effect in the $\mathrm{Adar}^{-1-}, \mathrm{Adarb}^{-1-}$ double-knockout embryos. Of these, the miR-30 cluster, miR-32, and miR-153 and their predicted target synapsin II are particularly interesting. It is exceptional that a group of five miRNAs that all target the same mRNA are deregulated. Synapsin II is associated with synaptic vesicles, and SNPs in this gene have already been linked to psychiatric disorders (Bogen et al. 2011).

The abundance of editing events detected in our embryonic samples is very low and never exceeds $6 \%$. However, the high read number and the consistent changes observed in the two knockout genotypes allowed us to reliably detect A-to-G transitions. The fact that we were also able to detect the previously reported miRNA editing events also shows the reliability of the applied method. Besides the already known editing events, we were able to detect six novel editing sites in miRNAs. Because we limited our analysis of A-to-G transitions to miRNAs with a minimal read depth of 100 or higher, it is conceivable that more editing events do exist in the mouse miRNA repertoire. In fact, we find evidence for additional editing events when miRNAs with lower read coverage are also considered. It is also interesting to note that ADARB1 is responsible for the majority of editing events in miRNAs. In at least two cases, we could observe an increase in editing in the absence of ADARB1. This finding would be consistent with the idea that some miRNAs are protected from being edited by ADAR by being bound to ADARB1. A similar finding has been made when editing events in SINEs were studied in the presence or absence of ADARs. Also, some sites in SINEs showed increased editing in the absence of ADARB1, suggesting that some sites might be protected from editing by ADARB1 (Riedmann et al. 2008).

The biological consequences of the observed editing events are hard to interpret. First, editing in the miRNAs does not correlate with their up-regulation or down-regulation. Second, $\sim 50 \%$ of the identified miRNAs show an editing event outside of their seed region. Third, and most important, the low editing levels detected here will most likely not result in a significant change in regulation of novel target mRNAs to which the edited miRNAs might be redirected. Nonetheless, it is known that overall RNA editing is very low during the early stages of embryonic development studied here. Therefore, targets identified here might very well become biologically relevant at later developmental stages. Since Adarb $1^{-1-}$ mice are viable and only exhibit mild phenotypes when rescued with a pre-edited Gria2 allele, it is possible that some of the observed ADARB1-dependent editing events in miRNAs lead to the observed phenotypic changes (Horsch et al. 2011). In any case, it is not likely that lack of the low numbers of editing events detected in miRNAs would be responsible for the observed embryonic lethality of $\mathrm{Adar}^{-1-}$ mice (Hartner et al. 2004; Wang et al. 2004). It is also worth considering that the majority of effects both on miRNA abundance and miRNA sequence change can be equally observed in Adarb $1^{-1-}$ and Adar $^{-1-}$, Adarb $1^{-1-}$ embryos. Because Adarb1 $1^{-/-}$ mice are viable well beyond the developmental stages investigated here, an indirect, secondary effect on miRNAs induced by loss of ADAR (which eventually leads to embryonic lethality) appears highly unlikely.

\section{Genome Research}

www.genome.org 


\section{Methods}

\section{Mice}

Adarb1 $1^{-1-}$ and Adar $^{-1-}$, Adarb1 ${ }^{-1-}$ knockout mice were a kind gift of Peter Seeburg. These transgenic mice are in an SV129 background, which was also used as the reference strain in our study. Mice were bred in our facility animal house. Pregnant mice were euthanized at 11.5 days postcoitum (dpc). Embryos were collected in 1× PBS and genotyped for Adar, Adarb1, and a Y-chromosomal marker (Brusa et al. 1995; Hartner et al. 2004).

\section{RNA extraction and miRNA cloning}

Female mouse embryos were dissected on E11.5 and homogenized, and total RNA was extracted using peqGOLD TriFast reagent according to the manufacturer's instructions (PEQLAB Biotechnologie $\mathrm{GmbH}$ ). DNA for genotyping was extracted from the remaining amnions, and sex determination was performed as described (Lambert et al. 2000). Total RNA was enriched for small RNAs (<200 nt) using the mirVana miRNA Isolation Kit (Ambion, Applied Biosystems). Small RNAs were 5'-end-labeled with $20 \mu \mathrm{Ci}$ $\left[\gamma^{-32} \mathrm{P}\right]$ ATP using T4 polynucleotide kinase (Fermentas). Radiolabeled RNA was separated on 15\% 19:1 AA:bisAA gels (8 M urea in $1 \times$ TBE) and exposed to X-ray films. RNA bands between 19 and 25 nt were cut out and eluted in $400 \mu \mathrm{L}$ of elution buffer $(500 \mathrm{mM}$ $\mathrm{NH}_{4} \mathrm{OAc}, 0.2 \%$ SDS, $100 \mathrm{mM}$ EDTA) overnight at room temperature. Eluted samples were purified over Sephadex G-25 spincolumns and precipitated. The size-selected RNA was ligated to a 5'-pre-adenylated and 3'-blocked 3'-end adapter (5'-Appp-UCG UAUGCCGUCUUCUGCUUGUidT- ${ }^{\prime}$ ') using truncated T4 RNA Ligase 2 (New England Biolabs) with the supplied buffer and a final concentration of 20\% PEG 6000. Ligations were incubated for $6 \mathrm{~h}$ at room temperature followed overnight by $4^{\circ} \mathrm{C}$.

Ligation products were purified on a $10 \%$ denaturing PAGE next to radiolabeled markers and ligated to the $5^{\prime}$ adapter ( $5^{\prime}$-GUU CAGAGUUCUACAGUCCGACGAUC-3') using T4 RNA Ligase 1 (New England Biolabs) with the supplied buffer and 20\% PEG. Again, the ligation product was purified on a denaturing PAGE. The final product was reverse-transcribed using RevertAid H Minus Reverse Transcriptase (Fermentas) using RT primer 5'-CAAGCAG AAGACGGCATACGA-3' following the manufacturer's instructions. The cDNA was used as template for 15 cycles of PCR amplification (forward primer: 5'-AATGATACGGCGACCACCGACA GGTTCAGAGTTCTACAGTCCGA-3'; reverse primer: 5'-CAAGC AGAAGACGGCATACGA3') using Phusion High-Fidelity DNA Polymerase (Finnzymes) according to the manufacturer's protocol. PCR products were separated on a nondenaturing 6\% AA TBE gel and stained with ethidium bromide in $1 \times$ TBE. Bands of $\sim 100 \mathrm{bp}$ corresponding to the final mature miRNA library were cut out and eluted in $400 \mu \mathrm{L}$ of elution buffer $\left(0.5 \mathrm{M} \mathrm{NH}_{4} \mathrm{OAc}, 1 \mathrm{mM}\right.$ EDTA at $\mathrm{pH}$ 8.1) overnight at room temperature. DNA was precipitated and subsequently resuspended in $10 \mu \mathrm{L}$ of $1 \times \mathrm{TE}$.

\section{Sequencing and clipping of reads}

Completed libraries were quantified with the Agilent Bioanalyzer dsDNA 1000 assay kit and Agilent QPCR NGS library quantification kit. Cluster generation and sequencing were performed using the Illumina Genome Analyzer (GA) IIx system according to the manufacturer's guidelines. After sequencing at a read length of $36 \mathrm{bp}$, adaptor sequences were removed using Cutadapt (Martin 2011).

\section{Mapping to mature miRNA sequences}

The mapping of the clipped reads to the reference (here mature miRNA sequences; release 16 of miRBase) (Kozomara and Griffiths-
Jones 2011) was performed with NextGenMap (FJ Sedlazeck, S Tauber, GB Ewing, and A von Haeseler, unpubl.), which computes a banded Smith-Waterman alignment (Smith and Waterman 1981) and uses a graphic card to speed up calculations.

A read is called a "putatively mapped read" if at least two subsequences of $10 \mathrm{bp}$ are identical to the reference and occur close to each other. For all regions in the reference where a read putatively maps, we compute a banded Smith-Waterman alignment (allowing for maximal 10 consecutive insertion or deletions) and select the region(s) with the highest alignment score. The actual alignment is calculated for these regions. If multiple regions provide the same highest score, the alignment is computed for each region. If the alignment of a mapped read has more then two substitutions, the read is excluded from a further analysis. We discarded reads that mapped to the minus strand of the annotated miRNA data set, since those are most probably due to annotation errors. Since the cloning protocol for miRNAs is strand-specific, reversely mapped miRNAs are also not expected.

\section{Identification of significant editing events}

$\mathrm{A} \chi^{2}$ test was used to identify putative editing events. Because Ato-G editing events are of interest, the test was applied for each A in the mature miRNA sequences taken from miRBase. The number of expected A's was estimated by the overall perfect match rate over all alignments. The number of expected G's was estimated by the number of observed substitutions (besides $A$ to $G$ ) over all alignments. The multiple tests problem was addressed by using Benjamini and Hochberg's method "fdr" (Benjamini and Hochberg 1995). Events that were significant in at least two out of three replicates were considered as relevant and used for further analysis.

\section{Microarray analysis}

Total RNA of wild-type, Adarb1 $1^{-/-}$, and Adar $^{-/-}$, Adarb1 $^{-1-}$ E11.5 embryos was isolated as described and sent for microarray analysis to Miltenyi Biotec $\mathrm{GmbH}$. The samples were analyzed using one $8 \times 60 \mathrm{~K}$ Agilent Whole Mouse Genome Microarray (one color) for each genotype. Gene-ratio lists were calculated by dividing the sample signal intensity by control (WT) signal intensity.

\section{Target gene predictions}

mmu-miR target genes were predicted by the TargetScan algorithm from TargetScanMouse Version 5.2 (http://www.targetscan.org/) using the default settings.

\section{Data access}

Illumina reads for 21-23-nt-long RNAs as well as the microarray data for wild-type, Adarb1 $1^{-1-}$, and $\mathrm{Adar}^{-1-}$, Adarb1 $1^{-1-}$ deficient embryos have been submitted to the NCBI Gene Expression Omnibus (GEO) (http://www.ncbi.nlm.nih.gov/geo/) under accession number GSE34626.

\section{Acknowledgments}

We thank Miya Higuchi and Peter Seeburg (MPI, Heidelberg) for the kind gift of Adarb1 $1^{-1-}$ and Adar ${ }^{-1-}$, Adarb1 $1^{-1-}$ knockout mice. This work was supported by the Austrian Science Foundation grant number F43 to M.F.J. C.V. was supported by Doctoral Program W1207 of the Austrian Science Foundation. S.T. and F.J.S. were supported by the WWTF to A.v.H. 


\section{References}

Barraud P, Allain FH. 2012. ADAR proteins: Double-stranded RNA and Z-DNA binding domains. Curr Top Microbiol Immunol 353: 35-60.

Bartel DP. 2004. MicroRNAs: Genomics, biogenesis, mechanism, and function. Cell 116: 281-297.

Benjamini Y, Hochberg Y. 1995. Controlling the false discovery rate: a practical and powerful approach to multiple testing. J R Stat Soc Ser B Methodol 57: 289-300.

Blow MJ, Grocock RJ, van Dongen S, Enright AJ, Dicks E, Futreal PA, Wooster R, Stratton MR. 2006. RNA editing of human microRNAs. Genome Biol 7: R27. doi: 10.1186/gb-2006-7-4-r27.

Bogen IL, Jensen V, Hvalby O, Walaas SI. 2011. Glutamatergic neurotransmission in the synapsin I and II double knock-out mouse. Semin Cell Dev Biol 22: 400-407.

Brusa R, Zimmermann F, Koh D, Feldmeyer D, Gass P, Seeburg P, Sprengel R. 1995. Early-onset epilepsy and postnatal lethality associated with an editing-deficient GluR-B allele in mice. Science 270: 1677-1680.

Burns C, Chu H, Rueter S, Hutchinson L, Canton H, Sanders-Bush E, Emeson R. 1997. Regulation of serotonin-2C receptor G-protein coupling by RNA editing. Nature 387: 303-308.

Chiang HR, Schoenfeld LW, Ruby JG, Auyeung VC, Spies N, Baek D, Johnston WK, Russ C, Luo S, Babiarz JE, et al. 2010. Mammalian microRNAs: experimental evaluation of novel and previously annotated genes. Genes Dev 24: 992-1009.

Eggington JM, Greene T, Bass BL. 2011. Predicting sites of ADAR editing in double-stranded RNA. Nat Commun 2: 319. doi: 10.1038/ncomms1324.

Friedman RC, Farh KK, Burge CB, Bartel DP. 2009. Most mammalian mRNAs are conserved targets of microRNAs. Genome Res 19: 92-105.

Gallo A, Locatelli F. 2012. ADARs: allies or enemies? The importance of A-toI RNA editing in human disease: from cancer to HIV-1. Biol Rev Camb Philos Soc 87: 95-110.

Hartner JC, Schmittwolf C, Kispert A, Muller AM, Higuchi M, Seeburg PH. 2004. Liver disintegration in the mouse embryo caused by deficiency in the RNA-editing enzyme ADAR1. J Biol Chem 279: 4894-4902.

Hartner JC, Walkley CR, Lu J, Orkin SH. 2009. ADAR1 is essential for the maintenance of hematopoiesis and suppression of interferon signaling. Nat Immunol 10: 109-115.

Heale BS, Keegan LP, McGurk L, Michlewski G, Brindle J, Stanton CM, Caceres JF, O'Connell MA. 2009. Editing independent effects of ADARs on the miRNA/siRNA pathways. EMBO J 28: 3145-3156.

Higuchi M, Maas S, Single F, Hartner J, Rozov A, Burnashev N, Feldmeyer D, Sprengel R, Seeburg P. 2000. Point mutation in an AMPA receptor gene rescues lethality in mice deficient in the RNA-editing enzyme ADAR2. Nature 406: $78-81$.

Hoopengardner B, Bhalla T, Staber C, Reenan R. 2003. Nervous system targets of RNA editing identified by comparative genomics. Science $\mathbf{3 0 1}$ : 832-836.

Horsch M, Seeburg PH, Adler T, Aguilar-Pimentel JA, Becker L, Calzada-Wack J, Garrett L, Gotz A, Hans W, Higuchi M, et al. 2011. Requirement of the RNA-editing enzyme ADAR2 for normal physiology in mice. J Biol Chem 286: 18614-18622.

Hu S, Huang M, Li Z, Jia F, Ghosh Z, Lijkwan MA, Fasanaro P, Sun N, Wang X, Martelli F, et al. 2010. MicroRNA-210 as a novel therapy for treatment of ischemic heart disease. Circulation (Suppl) 122: S124-S131.

Kawahara Y, Zinshteyn B, Chendrimada TP, Shiekhattar R, Nishikura K. 2007a. RNA editing of the microRNA-151 precursor blocks cleavage by the Dicer-TRBP complex. EMBO Rep 8: 763-769.

Kawahara Y, Zinshteyn B, Sethupathy P, Iizasa H, Hatzigeorgiou AG, Nishikura K. 2007b. Redirection of silencing targets by adenosine-toinosine editing of miRNAs. Science 315: 1137-1140.

Kawahara Y, Megraw M, Kreider E, Iizasa H, Valente L, Hatzigeorgiou AG, Nishikura K. 2008. Frequency and fate of microRNA editing in human brain. Nucleic Acids Res 36: 5270-5280.

Kleinberger Y, Eisenberg E. 2010. Large-scale analysis of structural, sequence and thermodynamic characteristics of A-to-I RNA editing sites in human Alu repeats. BMC Genomics 11: 453. doi: 10.1186/ 1471-2164-11-453.

Kozomara A, Griffiths-Jones S. 2011. miRBase: integrating microRNA annotation and deep-sequencing data. Nucleic Acids Res (suppl 1) 39: D152-D157.

Lambert J-F, Benoit BO, Colvin GA, Carlson J, Delville Y, Quesenberry PJ. 2000. Quick sex determination of mouse fetuses. J Neurosci Methods 95: 127-132.

Landgraf P, Rusu M, Sheridan R, Sewer A, Iovino N, Aravin A, Pfeffer S, Rice A, Kamphorst AO, Landthaler M, et al. 2007. A mammalian microRNA expression atlas based on small RNA library sequencing. Cell 129: 14011414.

Levanon E, Eisenberg E, Yelin R, Nemzer S, Hallegger M, Shemesh R, Fligelman Z, Shoshan A, Pollock S, Sztybel D. 2004. Systematic identification of abundant A-to-I editing sites in the human transcriptome. Nat Biotechnol 22: 1001-1005.

Li J, Levanon E, Yoon J, Aach J, Xie B, Leproust E, Zhang K, Gao Y, Church G. 2009. Genome-wide identification of human RNA editing sites by parallel DNA capturing and sequencing. Science 324: $1210-1213$.

Li M, Wang IX, Li Y, Bruzel A, Richards AL, Toung JM, Cheung VG. 2011. Widespread RNA and DNA sequence differences in the human transcriptome. Science 333: 53-58.

Martin M. 2011. Cutadapt removes adapter sequences from highthroughput sequencing reads. EMBnet.journal 17. http://journal. embnet.org/index.php/embnetjournal/article/view/200.

Nishikura K. 2010. Functions and regulation of RNA editing by ADAR deaminases. Annu Rev Biochem 79: 321-349.

Ohlson J, Pedersen JS, Haussler D, Öhman M. 2007. Editing modifies the GABAA receptor subunit $\alpha 3$. RNA 13: 698-703.

Riedmann E, Schopoff S, Hartner J, Jantsch M. 2008. Specificity of ADAR-mediated RNA editing in newly identified targets. RNA 14: 11101118.

Shen WF, Hu YL, Uttarwar L, Passegue E, Largman C. 2008. MicroRNA-126 regulates HOXA9 by binding to the homeobox. Mol Cell Biol 28: 46094619.

Smith TF, Waterman MS. 1981. Identification of common molecular subsequences. J Mol Biol 147: 195-197.

Sommer B, Köhler M, Sprengel R, Seeburg PH. 1991. RNA editing in brain controls a determinant of ion flow in glutamate-gated channels. Cell 67: 11-19.

Stefl R, Oberstrass FC, Hood JL, Jourdan M, Zimmermann M, Skrisovska L, Maris C, Peng L, Hofr C, Emeson RB, et al. 2010. The solution structure of the ADAR2 dsRBM-RNA complex reveals a sequence-specific readout of the minor groove. Cell 143: 225-237.

Wahlstedt H, Daniel C, Ensterö M, Öhman M. 2009. Large-scale mRNA sequencing determines global regulation of RNA editing during brain development. Genome Res 19: 978-986.

Wang Q, Miyakoda M, Yang W, Khillan J, Stachura DL, Weiss MJ, Nishikura K. 2004. Stress-induced apoptosis associated with null mutation of ADAR1 RNA editing deaminase gene. J Biol Chem 279: 4952-4961.

Wong S, Sato S, Lazinski D. 2001. Substrate recognition by ADAR1 and ADAR2. RNA 7: 846-858.

Wu D, Lamm AT, Fire AZ. 2011. Competition between ADAR and RNAi pathways for an extensive class of RNA targets. Nat Struct Mol Biol 18: 1094-1101.

Yang W, Chendrimada TP, Wang Q, Higuchi M, Seeburg PH, Shiekhattar R, Nishikura K. 2006. Modulation of microRNA processing and expression through RNA editing by ADAR deaminases. Nat Struct Mol Biol 13: 1321.

Zhang Z-W, Zhang L-Q, Ding L, Wang F, Sun Y-J, An Y, Zhao Y, Li Y-H, Teng C-B. 2011. MicroRNA-19b downregulates insulin 1 through targeting transcription factor NeuroD1. FEBS Lett 585: 2592-2598.

Received October 6, 2011; accepted in revised form February 1, 2012.

1476 Genome Research

www.genome.org 


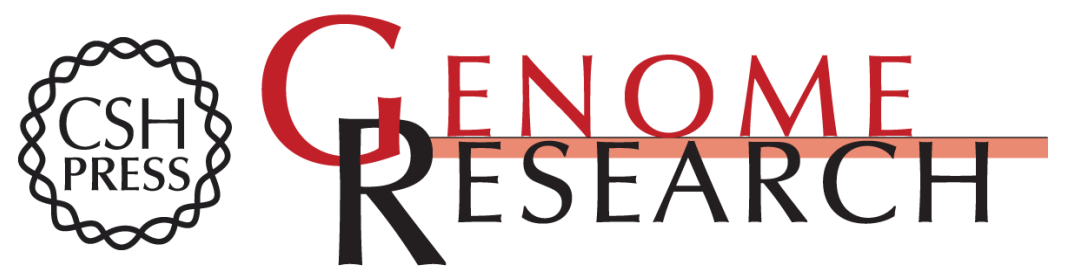

\section{Adenosine deaminases that act on RNA induce reproducible changes in abundance and sequence of embryonic miRNAs}

Cornelia Vesely, Stefanie Tauber, Fritz J. Sedlazeck, et al.

Genome Res. 2012 22: 1468-1476 originally published online February 6, 2012

Access the most recent version at doi:10.1101/gr.133025.111

\section{Supplemental Material \\ Related Content \\ References \\ Open Access \\ Creative Commons \\ License}

http://genome.cshlp.org/content/suppl/2012/02/06/gr.133025.111.DC1

Effects of ADARs on small RNA processing pathways in C. elegans M. Bryan Warf, Brent A. Shepherd, W. Evan Johnson, et al. Genome Res. August , 2012 22: 1488-1498 Systematic identification of edited microRNAs in the human brain

Shahar Alon, Eyal Mor, Francois Vigneault, et al.

Genome Res. August , 2012 22: 1533-1540 A-to-l editing of microRNAs in the mammalian brain increases during development Ylva Ekdahl, Hossein Shahrabi Farahani, Mikaela Behm, et al.

Genome Res. August , 2012 22: 1477-1487

This article cites 41 articles, 16 of which can be accessed free at:

http://genome.cshlp.org/content/22/8/1468.full.html\#ref-list-1

Articles cited in:

http://genome.cshlp.org/content/22/8/1468.full.html\#related-urls

Freely available online through the Genome Research Open Access option.

This article is distributed exclusively by Cold Spring Harbor Laboratory Press for the first six months after the full-issue publication date (see

http://genome.cshlp.org/site/misc/terms.xhtml). After six months, it is available under a Creative Commons License (Attribution-NonCommercial 3.0 Unported License), as described at http://creativecommons.org/licenses/by-nc/3.0/.

Receive free email alerts when new articles cite this article - sign up in the box at the top right corner of the article or click here.

\section{Affordable, Accurate Sequencing.}




\section{Affordable, Accurate} Sequencing.

To subscribe to Genome Research go to: https://genome.cshlp.org/subscriptions

(C) 2012, Published by Cold Spring Harbor Laboratory Press 